\title{
Association of serum Ninjurin2 levels with neurologic damage and postherpetic neuralgia occurrence: an observational cohort study in chinese herpeszoster patients
}

\author{
Guozhuan Zhang ${ }^{1}$, Yingjiao Sun ${ }^{1}$, Lei Wang ${ }^{1}$, Hui Tian² and Lishuang Liang ${ }^{1}$ \\ ${ }^{1}$ Department of Pain Management, Qilu Hospital of Shandong University, Jinan, Shandong, China \\ ${ }^{2}$ Department of Thoracic Surgery, Qilu Hospital of Shandong University, Jinan, Shandong, China \\ Correspondence to: Lishuang Liang, email: IIsh_117@163.com \\ Hui Tian, email: tianhuijinan@126.com
}

Keywords: Ninjurin2, neurologic damage, herpes zoster, postherpetic neuralgia occurrence

Received: February 21, $2017 \quad$ Accepted: March 23, $2017 \quad$ Published: May 04, 2017

Copyright: Zhang et al. This is an open-access article distributed under the terms of the Creative Commons Attribution License 3.0 (CC BY 3.0), which permits unrestricted use, distribution, and reproduction in any medium, provided the original author and source are credited.

\section{ABSTRACT}

Objectives: Postherpetic neuralgia(PHN) is the most common complication of herpeszoster ( $\mathrm{HZ}$ ) infection. The study aimed to explore whether serum Ninjurin2 (for nerve injury-induced protein 2, NINJ2), a novel neurologic damage related protein, is associated with nerve injury and the occurrence of PHN.

Results: Seventy-four of the eighty patients completed the study. On the 7th day of AHN, the patients had significantly higher values of NINJ2, cold-sense dispersion $(\Delta C S)$, warm-sense dispersion ( $\Delta W S$ ), cold-pain dispersion ( $\Delta C P$ ), heatpain dispersion ( $\triangle \mathrm{HP}$ ) and NRS score compared to controls. Six months after herpes, thirty four patients developed PHN. The values of serum NINJ2, $\triangle C S, \triangle W S, \triangle C P$ and $\triangle H P$ in PHN patients remained higher than in the controls and the patients who did not develop PHN. The PHN patients had significantly lower values of serumNINJ2 than patients who did not develop PHN in the period of AHN. With partial correlations and multivariable logistic regressions, serum NINJ2 level was strongly predictive of PHN.

Materials and Methods: Eighty individuals with acute herpetic neuralgia (AHN) and 60 controls were recruited. The following nerve injury testing was performed on all of the patients including: the numerical rating scale (NRS) test, quantitative sensory testing (QST), and the investigation of serum NINJ2 levels on the 7th day of AHN; in addition, all of the patients repeated all of the items at 6 months after herpes.

Conclusions: The serum NINJ2 levels are associated with nerve injury, particularly with nerve recovery, and may be predictors of PHN occurrence.

\section{INTRODUCTION}

Herpes zoster and postherpetic neuralgia

Zoster results from the reactivation of endogenous varicella zoster virus (VZV), which persisted in the latent form within sensory ganglia following varicella $[1,2]$. Herpes zoster(HZ) typically begins with unilateral pain that persists for several days before the appearance of a rash [3]. It may manifest as throbbing pain, stabbing pain, burning pain, lancinating pain, and other types of pain, and is consistent with a skin lesion. The pain may also occur accompanied by the rash or after the lesion heals [4]. Acute herpetic neuralgia (AHN) is defined as pain that occurs within 30 days after rash onset [5]. Postherpetic
neuralgia(PHN) is the most common complication and is usually defined as pain lasting beyond 6 months after the onset of zoster $[6,7]$.

Epidemiology and mechanism of PHN

In this study, PHN was defined as neuralgia lasting $\geq 6$ months [8]. Risk factors for PHN include increasing age, hyperglycemia, the presence of prodromal pain, the extent and severity of rash, and the severity of acute $\mathrm{HZ}$ pain $[9,10]$. An age $\geq 50$ years is more predictive of an occurrence of PHN if the patient was treated early with antiviral medications. There are other less replicated risk factors for PHN, such as female gender, location in the ophthalmic branch of the trigeminal nerve, greater 
neurosensory disturbance, and psychosocial distress [11, 12]. PHN can have a tremendous negative effect on quality of life due to its severe and durable pain. In population-based studies, zoster has been determined to be a risk factor for the development of major depression, and PHN has been reported to be the most common cause of suicide because of pain in persons aged 70 years and older [13]. The mechanism of PHN is still poorly understood. Thus, it is necessary to study the potential cause of PHN occurrence and to take related actions to prevent PHN.

\section{Ninjurin2 and nerve injury}

Ninjurin2(for nerve injury induced protein 2, NINJ2) is a homolog of ninjurin1(for nerve injury induced protein 1, NINJ1), a homophilic cellular adhesion molecule, that was previously isolated as a gene induced in Schwann cells after nerve injury [14]. NINJ2 mRNA was detected at low levels in normal nerves but was highly upregulated after nerve injury and reached peak levels 7-14 days after injury [14]. NINJ2 is upregulated in Schwann cells in the distal nerve segment after peripheral nerve injury, and it promotes neurite outgrowth from dorsal root ganglion neurons (DRG neurons) via NINJ2-mediated homophilic cellular interaction between neuronal axons and Schwann cells [14].

The disease course from $\mathrm{HZ}$ to $\mathrm{PHN}$ is a continuous process, and nerve injury and nerve recovery runs through the course. Thus, we propose that NINJ2 may be involved in the occurrence of PHN, and may help to explore the mechanism and predict the occurrence of PHN.

\section{RESULTS}

\section{Study population}

Of the 80 patients enrolled, 74 patients completed the study. Reasons for withdrawal included heart attacks $(n=2)$, cerebrovascular disease emergency $(n=2)$, death $(n=1)$, and unexplained reasons $(n=1)$.

The clinical characteristics of the subjects in all groups are listed in Table 1. The patient group and controls showed no significant differences with regard to gender, age, DM, hypertension, or CAD $(p>0.05)$.

\section{Nerve injury and NINJ2 level testing}

The $\Delta \mathrm{CS}, \Delta \mathrm{WS}, \Delta \mathrm{CP}, \Delta \mathrm{HP}, \mathrm{NRS}$ score, and serum NINJ2 levels were significantly greater in the patient group compared with the control group ( $p<0.001$, Table 1).

Thirty-four patients developed PHN at 6 months after the rash in our follow-up. Age and the blood glucose level on the 7th day after the rash were significantly higher in the PHN patients compared to patients who did not develop PHN ( $p<0.05$, Table 1$)$, but the gender, percentages of hypertension and coronary heart disease of both the PHN patients and non-PHN patients were not significantly different from the control $(p>0.05)$. On the 7 th day after the rash, the distributions of all types of temperature sensory data and the NRS score were not significantly different between the PHN patients and nonPHN patients ( $p>0.05$, Table 1$)$, but both of these groups dramatically differed from the controls $(p<0.001$, Table 1$)$. The serum NINJ2 levels were significantly greater in PHN patients and non-PHN patients compared with the controls, with the highest levels observed in non-PHN patients $(1969.97 \pm 230.02,739.21 \pm 109.94$ and $439.50 \pm 77.84 \mathrm{pg}$ ml-1, respectively, Table 1) on the 7 th day after the rash.

Six months after herpes, the $\Delta \mathrm{CS}, \Delta \mathrm{WS}, \Delta \mathrm{CP}$, $\triangle H P$, and NRS score of the PHN patients were greater than those of the controls and the non-PHN patients $(p<0.001$, Table 1). However, for the non-PHN patients, the $\Delta \mathrm{CS}, \Delta \mathrm{WS}, \Delta \mathrm{CP}$ decreased to normal levels, but $\Delta \mathrm{HP}$ and the NRS score remained higher than in the controls $(p<0.05, p<0.001$, Table 1). The serum NINJ2 levels were significantly greater in PHN patients compared with controls and non-PHN patients $(841.91 \pm 83.15,420.47 \pm 57.2$ and $348.76 \pm 33.47$ pg ml-1, respectively, Table 1 ).

For the PHN patients, the $\Delta \mathrm{CS}, \Delta \mathrm{WS}, \Delta \mathrm{CP}, \Delta \mathrm{HP}$, and NRS score were significantly greater than in the controls and were similar to those observed during AHN $(p<0.001$, Figure 1). The serum NINJ2 levels of the PHN patients after 6 months were even greater than those on the 7th day after the rash $(841.91 \pm 83.15,739.21 \pm 109.94 \mathrm{pg}$ ml-1,respectively, Figure 1).For the non-PHN patients, the $\Delta \mathrm{CS}, \Delta \mathrm{WS}, \Delta \mathrm{CP}, \Delta \mathrm{HP}, \mathrm{NRS}$ score, and serum NINJ2 levels were significantly greater than in the controls on the 7 th day after the $\operatorname{rash}(p<0.001$, Figure 2$), \Delta \mathrm{HP}$ and NRS score maintained still higher levels than controls after 6 months ( $p<0.001, p<0.05$, Figure 2) whereas $\Delta \mathrm{CS}, \Delta \mathrm{WS}$, $\triangle \mathrm{CP}$ and serum NINJ2 levels returned to normal levels after 6 months compared with controls ( $p>0.05$, Figure 2$)$.

\section{Correlation of PHN occurrence with clinical parameters and NINJ2}

In all patients, after controlling for gender, hypertension, and coronary heart disease, PHN occurrence showed significant correlations with the lower serum NINJ2 levels, presence of diabetes and older age on the 7-th day $(r=-0.404, p<0.001 ; r=0.277, p=0.022 ; r=0.279, p=$ $0.018)$, but does not appear to be correlated with NRS-7d, $\Delta \mathrm{CS}-7 \mathrm{~d}, \Delta \mathrm{WS}-7 \mathrm{~d}, \Delta \mathrm{CP}-7 \mathrm{~d}$ and $\Delta \mathrm{HP}-7 \mathrm{~d}(p=0.110-0.686)$ (Table 2). The higher NINJ2-6m level was significantly correlated with older age at baseline and higher NRS score, bigger $\Delta \mathrm{CS}, \Delta \mathrm{WS}, \Delta \mathrm{CP}$ and $\Delta \mathrm{HP}(r=0.283, p=0.019 ; r=$ $0.641, p<0.001 ; r=0.602, p<0.001 ; r=0.611, p<0.001$; $r=0.398, p=0.001 ; r=0.509, p<0.001$, respectively) (Table 3 ). In the multivariable logistic regression analysis adjusting for serum NINJ2 levels on the 7-th day after rash, age, and blood glucose, all of them were significantly associated with PHN occurrence $(\beta=-0.001, p<0.001$; $\beta=0.055, p=0.029 ; \beta=2.069, p=0.011$ ) (Table 4). In 
Table 1: Clinicopathological characteristics of the subjects

\begin{tabular}{|c|c|c|c|c|}
\hline Variables & Controls $(n=60)$ & All Patients $(n=74)$ & Non-PHN patients $(n=40)$ & PHN patients $(n=34)$ \\
\hline Male $(\%)$ & 43.3 & 45.9 & 44.1 & 47.5 \\
\hline Age (year) & $66.62 \pm 8.281$ & $66.14 \pm 10.00$ & $63.75 \pm 9.00$ & $68.94 \pm 10.52^{\star}$ \\
\hline $\mathrm{DM}(\%)$ & 16.7 & 16.2 & 5 & $29.4^{\wedge}$ \\
\hline HP $(\%)$ & 55 & 54 & 60 & 47.1 \\
\hline CAD $(\%)$ & 58.3 & 52.7 & 47.5 & 58.8 \\
\hline NRS-7d & $0.10 \pm 0.04$ & $5.35 \pm 0.29^{* * *}$ & $5.82 \pm 0.40^{* * *}$ & $4.95 \pm 0.4^{* * *}$ \\
\hline$\Delta \mathrm{CS}-7 \mathrm{~d}$ & $1.66 \pm 0.09$ & $6.12 \pm 0.14^{* * *}$ & $6.29 \pm 0.19^{* * *}$ & $6.09 \pm 0.17^{* * *}$ \\
\hline$\Delta \mathrm{WS}-7 \mathrm{~d}$ & $1.32 \pm 0.08$ & $5.05 \pm 0.1^{* * *}$ & $4.86 \pm 0.17^{* * *}$ & $5.21 \pm 0.12^{* * *}$ \\
\hline$\Delta \mathrm{CP}-7 \mathrm{~d}$ & $1.84 \pm 0.12$ & $3.44 \pm 0.09^{* * *}$ & $3.5 \pm 0.12^{* * *}$ & $3.39 \pm 0.12^{* * *}$ \\
\hline$\Delta \mathrm{HP}-7 \mathrm{~d}$ & $1.44 \pm 0.09$ & $2.17 \pm 0.07^{* * *}$ & $2.25 \pm 0.12^{* * *}$ & $2.10 \pm 0.09^{* * *}$ \\
\hline NINJ2-7d (pg ml-1) & $439.50 \pm 77.84$ & $1404.49 \pm 151.47^{* * *}$ & $1969.97 \pm 230.02^{* * *}$ & $739.21 \pm 109.94^{* \boldsymbol{\Delta} \Delta}$ \\
\hline NRS-6m & $0.083 \pm 0.28$ & $3.35 \pm 3.19^{* *}$ & $0.68 \pm 0.11^{* * *}$ & $6.5 \pm 0.30^{* * * \Delta \Delta}$ \\
\hline$\Delta \mathrm{CS}-6 \mathrm{~m}$ & $1.65 \pm 0.69$ & $3.7 \pm 2.25^{*}$ & $1.79 \pm 0.10$ & $5.95 \pm 0.13^{* * * \boldsymbol{\Lambda} \Lambda}$ \\
\hline$\Delta \mathrm{WS}-6 \mathrm{~m}$ & $1.35 \pm 0.57$ & $2.97 \pm 1.77^{* *}$ & $1.48 \pm 0.08$ & $4.72 \pm 0.15^{* * * \Delta \Delta}$ \\
\hline$\Delta \mathrm{CP}-6 \mathrm{~m}$ & $1.78 \pm 0.7$ & $2.37 \pm 0.67^{* *}$ & $1.97 \pm 0.07$ & $2.84 \pm 0.10^{* * * \boldsymbol{\Lambda} \Delta}$ \\
\hline$\Delta \mathrm{HP}-6 \mathrm{~m}$ & $1.43 \pm 0.70$ & $2.1 \pm 0.66^{* *}$ & $1.74 \pm 0.08^{*}$ & $2.52 \pm 0.10^{* * * \Delta \Delta}$ \\
\hline NINJ2-6m (pg ml-1) & $420.47 \pm 57.2$ & $575.34 \pm 43.74^{*}$ & $348.76 \pm 33.47$ & $841.91 \pm 83.15^{* * \Delta \Delta}$ \\
\hline
\end{tabular}

Abbreviations: DM, diabetes mellitus; HP, hypertension; CAD, coronary heart disease; NRS, numerical rating scale; $\Delta \mathrm{CS}$, $\Delta \mathrm{WS}, \Delta \mathrm{CP}, \Delta \mathrm{HP}$, the differences of threshold temperature of cold sense, warm sense, cold pain and heat pain between the two sides of the corresponding; NINJ2, nerve injury induced protein 2. N/A : Not available.

Values are given as mean \pm s.d.

$* P<0.05 ; * * P<0.01 ; * * * P<0.001$ compared with controls.

$\triangle P<0.05 ;{ }^{\wedge}{ }^{\Delta} P<0.001$ compared with non-PHN patients.

other word, the log odd of PHN occurrence increases 0.055 for one-year older patient, decreases 0.001 for one unit (pg/ $\mathrm{ml}$ ) increase of NINJ2 levels on the 7-th day after rash, or is 2.069 bigger in patients with diabetes than that in patients without diabetes, when adjusting for the other variables.

\section{DISCUSSION}

Previous studies have found that NINJ2 is associated with nerve injury in ischemic stroke [15]. Toshiyuki Araki and Jeffrey Milbrandt [14] found that
NINJ2 is expressed at low levels in the normal nerve but is highly upregulated after nerve injury. NINJ2 is associated with nerve injury and nerve recovery. The disease course from HZ to PHN is a continuous process, and nerve injury and nerve recovery run through this course. Thus, we detected the relationship between serum NINJ2 levels and PHN occurrence. Our current study is the first to report associations between serum NINJ2 levels and the parameters of nerve injury and nerve recovery and to detect the relationship between serum NINJ2 levels and PHN occurrence.

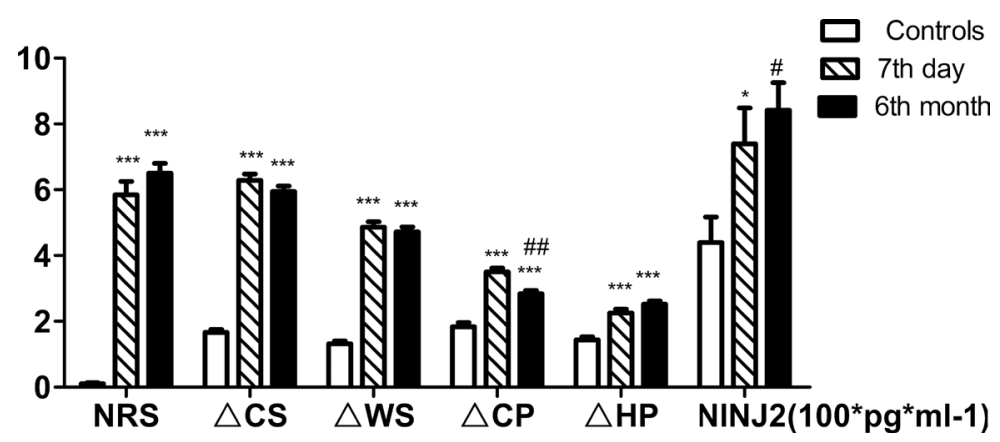

Figure 1: Changing of QST and serum NINJ2 levels of PHN patients. Abbreviations: NRS, numerical rating scale; $\Delta C S$, $\Delta \mathrm{WS}, \Delta \mathrm{CP}, \Delta \mathrm{HP}$, the differences of threshold temperature of cold sense, warm sense, coldpain and heat pain between the two sides of the corresponding; NINJ2, nerve injury induced protein 2 . Values are given as mean $\pm \mathrm{s}$. d. $* P<0.05 ; * * P<0.01 ; * * * P<0.001$ compared with control group; ${ }^{\#} P<0.05 ;{ }^{\#} P<0.001$ compared with 7 th day; One hundred times of NINJ2 values this chart shows are the actual value. 
In our study, serum NINJ2 levels increased to different degrees during AHN and PHN. The serum NINJ2 levels were significantly greater in PHN patients and non-PHN patients compared with the controls, accordance with the $\Delta \mathrm{CS}, \Delta \mathrm{WS}, \Delta \mathrm{CP}, \Delta \mathrm{HP}, \mathrm{NRS}$ score, with the highest NINJ2 levels observed in non-PHN patients on the 7 th day after the rash. During AHN, nerve injury occurred and the body began responding to this injury and nerve recovery began. Six months after herpes, for the PHN patients, the serum NINJ 2 levels remained higher the controls and even higher than those detected during AHN. However, for the non-PHN patients, the serum NINJ2 levels decreased to normal contrary to the $\Delta \mathrm{CS}, \Delta \mathrm{WS}, \Delta \mathrm{CP}$. The $\Delta \mathrm{HP}$ and NRS scores of non-PHN patients were more or less higher than control, but the NRS kept under 1. When PHN occurred, nerve injury and nerve recovery continued and lased for 6 months. But when $\mathrm{HZ}$ and nerve injury cured, nerve recovery slowed down to the normal condition. Accordantly, the serum NINJ2 levels were significantly greater in PHN patients compared with controls and non-PHN patients $(841.91 \pm$ $83.15,420.47 \pm 57.2$ and $348.76 \pm 33.47 \mathrm{pg} \mathrm{ml}-1$, respectively, Table 1). So NINJ2 levels were significantly correlated with clinical markers of nerve injury and nerve recovery during the process of PHN. Taken together, these findings suggested that serum NINJ2 levels may predict the occurrence of PHN

In our study, serum NINJ2 levels increased to different degrees during AHN and PHN. NINJ2 levels were significantly correlated with clinical markers of nerve injury and nerve recovery during the process of PHN. Taken together, these findings suggested that serum NINJ2 levels can predict the occurrence of PHN. Activation of latent Varicella zoster virus (VZV) causes generalized necrosis and cell death in the skin and within the nerve, root, and ganglion [16]. Previous studies also demonstrated damage in the dorsal root ganglia (DRG) as well as a loss of neurons in the affected spinal nerves and dorsal horn $[17,18]$. Nerve recovery occurs immediately following injury, but in human studies on sensory nerve injuries and their recovery, the precise pathophysiological mechanism and the extent of nerve injury are not known due to the heterogeneity of patients with regard to the type, degree, and age of the lesion, all of which affect recovery $[19,20]$. Because acute nerve damage is widespread in patients, no relationship was observed in the partial correlation analysis of the relationship between NINJ2 levels and the parameters of nerve injury on the 7 th day. However, after 6 months, partial correlation analysis showed that higher serum NINJ2 levels were significantly correlated with older age, higher NRS score, bigger $\Delta \mathrm{CS}, \Delta \mathrm{WS}, \Delta \mathrm{CP}$, and $\Delta \mathrm{HP}(r=0.283, p=0.019 ; r=0.641, p<0.001 ; r=$ $0.602, p<0.001 ; r=0.611, p<0.001 ; r=0.398, p=0.001$; $r=0.509, p<0.001$ ) (Table 3). Thus, serum NINJ2 levels were correlated with nerve injury and particularly with nerve recovery.

Following acute zoster, the patients were characterized with dermatomal areas of cutaneous scarring and sensory loss independent of PHN [21, 22]. The anatomical loss and injury of afferent neurons and fibers were consistent with the functional decrease in sensory perception within the affected dermatomes. In our study, the values of all types of temperature sensory data and the NRS score increased compared with controls, which indicated that peripheral sensory nerves suffered extensive damage. After 6 months, the $\triangle \mathrm{CS}, \Delta \mathrm{WS}, \Delta \mathrm{CP}$, and serum NINJ2 levels of the non-PHN patients significantly decreased, but $\triangle H P$ remained higher than normal. For the PHN patients, $\triangle \mathrm{CP}$ decreased but remained higher than normal, and the $\Delta \mathrm{CS}, \Delta \mathrm{WS}, \Delta \mathrm{HP}$, and NRS score of the PHN patients remained significantly higher than in the control group. The serum NINJ2 levels of the non- PHN

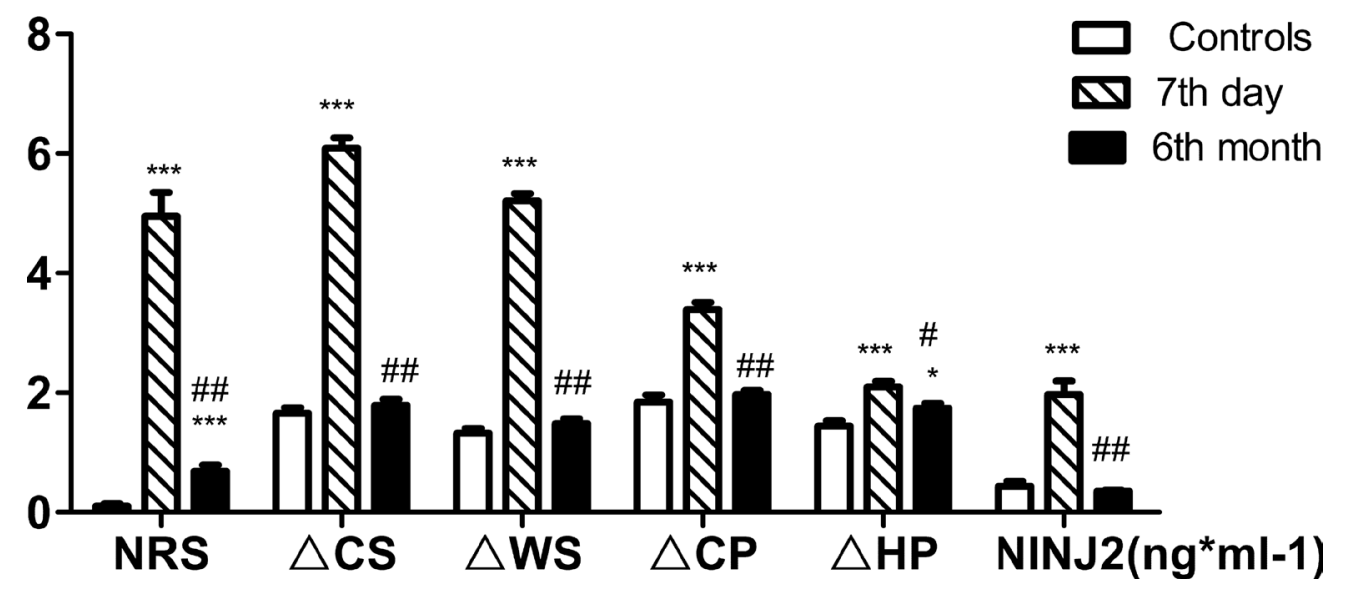

Figure 2: Changing of QST and serum NINJ2 levels of non-PHN patients. Abbreviations: NRS, numerical rating scale; $\Delta C S$, $\Delta \mathrm{WS}, \Delta \mathrm{CP}, \Delta \mathrm{HP}$, the differences of threshold temperature of cold sense, warm sense, coldpain and heat pain between the two sides of the corresponding; NINJ2, nerve injury induced protein 2 . Values are given as mean \pm s. d. $* P<0.05 ; * * P<0.01 ; * * * P<0.001$ compared with control group; ${ }^{P} P<0.05$; ${ }^{\#} P<0.001$ compared with 7 th day; One thousand times of NINJ2 values this chart shows are the actual value. 
Table 2: Partial correlations analysis between NINJ2 levels, PHN occurrence and parameters at the 7 th day of herpes

\begin{tabular}{|c|c|c|c|c|}
\hline & \multicolumn{2}{|c|}{ NINJ2-7d } & \multicolumn{2}{|c|}{ PHN occurrence } \\
\hline & Partial coefficient & $p$ value & Partial coefficient & $p$ value \\
\hline Age(year) & 0.067 & 0.575 & 0.279 & 0.018 \\
\hline DM & -0.05 & 0.0627 & 0.277 & 0.022 \\
\hline NINJ2-7d & & & -0.404 & $<0.001$ \\
\hline NRS-7d & 0.093 & 0.44 & 0.168 & 0.162 \\
\hline$\Delta \mathrm{CS}-7 \mathrm{~d}$ & -0.13 & 0.28 & 0.093 & 0.441 \\
\hline$\Delta \mathrm{WS}-7 \mathrm{~d}$ & -0.049 & 0.686 & -0.191 & 0.11 \\
\hline$\Delta \mathrm{CP}-7 \mathrm{~d}$ & -0.096 & 0.425 & 0.091 & 0.448 \\
\hline$\Delta \mathrm{HP}-7 \mathrm{~d}$ & -0.078 & 0.52 & 0.119 & 0.325 \\
\hline
\end{tabular}

Abbreviations: DM, diabetes mellitus; NINJ2, nerve injury induced protein 2, PHN, postherpetic neuralgia.

Table 3: Partial correlations analysis between NINJ2 levels and parameters after 6 months

\begin{tabular}{lcc}
\hline & \multicolumn{1}{c}{ NINJ2 } & p value \\
\cline { 2 - 3 } & Partial coefficient & 0.019 \\
Age(year) & 0.283 & $<0.001$ \\
NRS-6m & 0.641 & $<0.001$ \\
$\Delta$ CS-6m & 0.602 & $<0.001$ \\
$\Delta$ WS-6m & 0.611 & 0.001 \\
$\Delta$ CP-6m & 0.398 & $<0.001$ \\
$\Delta$ HP-6m & 0.509 & $<0.001$ \\
PHN & 0.707 & \\
\hline
\end{tabular}

Abbreviations: NRS, numerical rating scale; $\Delta \mathrm{CS}, \Delta \mathrm{WS}, \Delta \mathrm{CP}, \Delta \mathrm{HP}$, the differences of threshold temperature of cold sense, warm sense, cold pain and heat pain between the two sides of the corresponding; NINJ2, nerve injury induced protein 2; PHN, postherpetic neuralgia.

Table 4: Analysis and selection of variables affecting the PHN occurrence in multivariable logistic regression model

\begin{tabular}{cccc}
\hline Dependent variable & Independent variables & $\boldsymbol{\beta}$ & $\boldsymbol{P}$-value \\
\hline PHN & Age (year) & 0.055 & 0.029 \\
PHN & DM & 2.069 & 0.011 \\
PHN & NINJ2-7d & -0.001 & $<0.001$ \\
\hline
\end{tabular}

Abbreviations: DM, diabetes mellitus; NRS, numerical rating scale; NINJ2, nerve injury induced protein 2; PHN, postherpetic neuralgia.

$\beta$ : standard regression coefficient.

patients increased significantly compared to the levels observed on the 7 th day $(p<0.05)$, which suggested that NINJ2 levels were related with nerve recovery and repair.

Excess electrical activity of damaged peripheral nociceptive neurons is a major cause of acute zoster pain and of inflammatory and nociceptive pain in general. If the pain continues, then the electrical responses of dorsal horn nociceptive neurons that project to the brain may increase and cause sensitization, and PHN can occur. The important features of pain following nerve injury can be discerned from clinical observation [23]. In our study, 46 percent of HZ patients developed PHN. The well-defined risk factors for PHN in patients with $\mathrm{HZ}$ include older age, a high blood glucose level, presence of prodromal pain, the extent and severity of rash, and the severity of acute HZ pain [9].Specifically, both genetic and environmental influences contribute to the likelihood of developing PHN after nerve injury during AHN [23]. In our study, we found that the occurrence of PHN is significantly correlated with age, blood glucose level and serum NINJ2 levels using partial correlation analysis and multivariable linear regression analysis (Table 2, Table 4).

In this study, we found that serum NINJ2 levels were correlated with nerve recovery.NINJ2 is associated nerve repair when nerve system facing injury. In the early stages of the disease, more NINJ2 means more protection 
and potential repair for non- PHN subjects. HZ patients may not develop PHN when they exhibit high levels of serumNINJ2 during a AHN. In the patients who developed PHN, nerve recovery continued and the serum NINJ2 level remained relative high for a long time. These results suggest that serum NINJ2 levels may be a predictor of $\mathrm{PHN}$ in early stage.

A potential limitation of the present study was that prospective studies should provide further insight into the potential cause-effect relationships between preclinical markers and NINJ2 levels. There may be several other factors affecting the association between clinical markers and NINJ2, which requires additional studies.

\section{MATERIALS AND METHODS}

\section{Participants}

This research was approved by the Ethics Committee of Qilu Hospital. A total of 80 patients who were diagnosed with AHN within 7 days at the Department of Pain Management, Qilu Hospital, from January 2014 to June 2015,were enrolled for our research. 60 volunteers who were recruited as controls at the Department of Pain Management, Qilu Hospital, from January 2014 to June 2015. For all volunteers, neurological and routine blood parameters were assessed to meet the exclusion criteria. The research ethics boards at the participating centers approved the study protocol, and each participants were informed and provided their written informed consent before participating in the study. The study was performed according to the Helsinki Declaration.

Inclusion criteria for $\mathrm{HZ}$ patients were as follows: 1) Provided written informed consent; 2) $\geq 50$ years of age; 3) diagnosed with HZ within 7 days; 4) sufficient cognitive ability to be able to accurately assess their own state, including pain intensity, quality of life, and adverse reactions; and 5) no other form of neuralgia or nerve injury condition; Inclusion criteria for controls were as follows: 1) Provided written informed consent; 2) $\geq 50$ years of age; 3 ) sufficient cognitive ability to be able to accurately assess their own state, including pain intensity, and quality of life; 4) no form of neuralgia or nerve injury condition; and 5) an NRS score of $0-1$.

Exclusion criteria were as follows: 1) Severe disease of the liver or other organs; 2) a mental disorder that prevented them from evaluating themselves accurately; 3) any form of neuralgia or nerve injury condition; and 4) cerebral stroke or other form of cerebrovascular disease.

\section{Study design}

All of the participants were given a numerical rating scale (NRS) test, quantitative sensory testing (QST), and a 2-ml blood draw to test NINJ2 levels on the day of enrollment for the controls and on the 7th day of $\mathrm{HZ}$ for the patients. Six months after the rash, All patients repeated all of the items 6 months after the rash. The volunteers also were given same testing 6 months after the enrollment.

When enrolled, all the patients were given oral acyclovir ( Bangna, Xinda, Shandong, China) for 7 days, and analgesic therapies, including oral pregabalin (Lyrica, Pfizer, NY, America) and oxycodone (OxyContin, Mundipharma, Beijing, China). The start dosage of pregabalin was $150 \mathrm{mg} /$ day, and if there was no observed change of NRS, the dosage of pregabalin was increased to $300 \mathrm{mg} /$ day, until the maximal dose $600 \mathrm{mg} /$ day, and the start dosage oxycodone was $20 \mathrm{mg} /$ day, and the dosage increased according to pain assessment. If patients appeared adverse events including dizziness, somnolence, vertigo, dry mouth, constipation, edema, and so on, we would stop adding dosage and given corresponding therapies. The patients were administered antiviral and analgesic therapies during the intervening period. Two independent experts made the diagnosis of PHN according to the symptoms, characteristics of pain, QST and NRS score.

\section{Outcome measures}

\section{Baseline clinical characteristics}

The gender, age, history of illness (such as hypertension), and medication history were recorded.

\section{Pain intensity}

Pain intensity was assessed using NRS before study participation and at the follow-up visits with $0=$ no pain and $10=$ the worst pain imaginable. Patients dictated or lined out the score, which could describe their pain density.

\section{QST}

$\mathrm{HZ}$ and $\mathrm{PHN}$ involve nerve injury, and neuropathic pain (NP) is the predominant manifestation of the nerve injury. We diagnosed nerve injury and PHN based on QST in addition to neuropathic pain symptoms and signs. QST is a noninvasive procedure that is helpful in the assessment of the function of small $\mathrm{A} \delta$ and $\mathrm{C}$ nerve sensory fibers, which is hardly evidenced by other methods [24, 25]. The assessments were performed in a quiet, temperaturecontrolled room (mean across all tests: $23.4^{\circ} \mathrm{C}$ ) with a computerized thermal sensory analyzer (TSA) model TSAII (Medoc Inc., Ramat Yishai, Israel). QST was performed at two sites on the body: (1) the affected side and (2) the contralateral side (the mirror region). We detected coldsense (CS), warm-sense (WS), cold-pain (CP) and heatpain (HP) thresholds using the method of limits. Subjects were instructed to press are sponse button when a thermal sensation (either cold or warm) was first perceived for the 
CS and WS processes, which started from a resting neutral temperature of $32^{\circ} \mathrm{C}$ and gradually decreased or increased by $1^{\circ} \mathrm{C}$ per second $[25,26]$. Next, the computer recorded the threshold temperature and returned to the neutral temperature. The procedure was similar for $\mathrm{CP}$ and $\mathrm{HP}$, but the subjects may feel discomfort for these tests and they were instructed to press the response button immediately after they perceived the thermal sensation as painful. The differences in threshold temperature between the two sides of the body were recorded as the dispersions of QST for comparison and were recorded as $\triangle \mathrm{CS}, \Delta \mathrm{WS}, \Delta \mathrm{CP}, \Delta \mathrm{HP}$.

\section{Serum NINJ2 levels detected using enzyme- linked immune sorbent assay (ELISA)}

Serum NINJ2 levels were determined using an ELISA kit (Proteintech, Rosemont, USA) according to the manufacturer's instructions. The absorbance values were measured at $450 \mathrm{~nm}$ [27].

\section{PHN}

Two experts diagnosed that the patients were suffering from PHN 6 months after the rash. PHN is primarily a clinical diagnosis. A history of herpes zoster rash, followed by persistent pain (include throbbing pain, stabbing pain, burning pain, lancinating pain, and other types of pain) in the corresponding dermatome or adjoining area is typical [28].

\section{Statistical analysis}

Continuous data are presented as the mean $\pm \mathrm{SD}$. Categorical data were summarized as percentages. Oneway ANOVA of normally distributed continuous data was used to compare the differences among groups of subjects. Comparison of the prevalence of comorbid conditions were made using the $\chi^{2}$ test or Fisher's exact test if necessary. The correlation between two variables was assessed by Pearson or Spearman correlation coefficient analysis. After controlling for covariates, bivariate correlations underwent partial correlation analysis. Multivariable logistic regressions using the forward conditional method were used to determine the variables that were putative predictive factors (As mentioned before, age and diabetes are independent risk factors confirmed by other studies $[10,11])$. So age, diabetes and NINJ2-7d were added into multivariable logistic regression analysis as independent variables when PHN occurrence was dependent variable). All statistical analyses were performed using SPSS 12.0 software (SPSS Inc. Chicago, Illinois, America). $P$-values $<0.05$ were considered statistically significant.

\section{CONCLUSIONS}

In conclusion, the serum NINJ2 level is associated with nerve injury, and with nerve recovery in particular, and may be a predictor of PHN occurrence.

\section{ACKNOWLEDGMENTS AND FUNDING}

This work was supported by grants from the National Natural Science Foundation of China (81672292), the Natural Science Foundation of Shandong Province (ZR2013HM089), and Key research and development program of Shandong Province (2016GSF201159), two new fundings would be added if it is convenient.

\section{CONFLICTS OF INTEREST}

The authors declare no conflicts of interest.

\section{REFERENCES}

1. Weaver BA. The burden of herpes zoster and postherpetic neuralgia in the United States. J Am Osteopath Assoc. 2007; 107:S2-7.

2. Gnann JW, Whitley RJ. Clinical practice. Herpes zoster. N Engl J Med. 2002; 347:340-346.

3. Arvin AM. Varicella-zoster virus. Clin Microbiol Rev. 1996; 9:361-381.

4. Kanodia SK, Seth AK, Dixit AM. Dose related efficacy of gabapentin in acute herpetic neuralgia among geriatric patients. Indian J Dermatol. 2012; 57:362-365.

5. Jeon YH. Herpes Zoster and Postherpetic Neuralgia: Practical Consideration for Prevention and Treatment. Korean J Pain. 2015; 28:177-184.

6. Volpi A, Gross G, Hercogova J, Johnson RW. Current management of herpes zoster: the European view. Am J Clin Dermatol. 2005; 6:317-325.

7. Drolet M, Brisson M, Schmader KE, Levin MJ, Johnson R, Oxman MN, Patrick D, Blanchette C, Mansi JA. The impact of herpes zoster and postherpetic neuralgia on health-related quality of life: a prospective study. CMAJ. 2010; 182:1731-1736.

8. Liang L, Li X, Zhang G, Sun Y, Yu H, Jiao J. Pregabalin in the Treatment of Herpetic Neuralgia: Results of a Multicenter Chinese Study. Pain Med. 2015; 16:160-167.

9. Coen PG, Scott F, Leedham-Green M, Nia T, Jamil A, Johnson RW, Breuer J. Predicting and preventing postherpetic neuralgia: are current risk factors useful in clinical practice? Eur J Pain. 2006; 10:695-700.

10. Bosco D, Plastino M, De Bartolo M, Cristiano D, Ettore M, Zurlo G, Bosco F, Colica C, Tallarigo F, Fava A. Role of impaired glucose metabolism in the postherpetic neuralgia. Clin J Pain. 2013; 29:733-736.

11. Jung BF, Johnson RW, Griffin DRJ, Dworkin RH. Risk factors for postherpetic neuralgia in patients with herpes zoster. Neurology. 2004; 62:1545-1551.

12. Volpi A, Gatti A, Pica F, Bellino S, Marsella LT, Sabato AF. Clinical and psychosocial correlates of post-herpetic neuralgia. J Med Virol. 2008; 80:1646-1652. 
13. Chen MH, Wei HT, Su TP, Li CT, Lin WC, Chang WH, Chen TJ, Bai YM. Risk of depressive disorder among patients with herpes zoster: a nationwide population-based prospective study. Psychosom Med. 2014; 76:285-291.

14. Araki T, Milbrandt J. Ninjurin2, a novel homophilic adhesion molecule, is expressed in mature sensory and enteric neurons and promotes neurite outgrowth. J Neurosci. 2000; 20:187-195.

15. Wang X, Zhang J, Liu Y, Zhang Y. Relationship between nerve injury-induced protein gene 2 polymorphism and stroke in Chinese Han population. J Biomed Res. 2011; 25:287-291.

16. Dworkin RH, O'Connor AB, Backonja M, Farrar JT, Finnerup NB, Jensen TS, Kalso EA, Loeser JD, Miaskowski C, Nurmikko TJ, Portenoy RK, Rice ASC, Stacey BR, et al. Pharmacologic management of neuropathic pain: evidence-based recommendations. Pain. 2007; 132:237-251.

17. Philip A, Thakur R. Post herpetic neuralgia. J Palliat Med. 2011; 14:765-773.

18. Watson CP, Deck JH, Morshead C, Van der Kooy D, Evans RJ. Post-herpetic neuralgia: further post-mortem studies of cases with and without pain. Pain. 1991; 44:105-117.

19. Oaklander AL, Rissmiller JG, Gelman LB, Zheng L, Chang Y, Gott R. Evidence of focal small-fiber axonal degeneration in complex regional pain syndrome-I (reflex sympathetic dystrophy). Pain. 2006; 120:235-243.

20. Teerijoki-Oksa T, Jääskeläinen S, Forssell K, Virtanen A, Forssell H. An evaluation of clinical and electrophysiologic tests in nerve injury diagnosis after mandibular sagittal split osteotomy. Int J Oral Maxillofac Surg. 2003; 32:15-23.

21. Baron R, Saguer M. Postherpetic neuralgia. Are $\mathrm{C}$-nociceptors involved in signalling and maintenance of tactile allodynia? Brain. 1993; 116:1477-1496.
22. Mainka T, Malewicz NM, Baron R, Enax-Krumova EK, Treede RD, Maier C. Presence of hyperalgesia predicts analgesic efficacy of topically applied capsaicin $8 \%$ in patients with peripheral neuropathic pain. Eur J Pain. 2015; 116:1477-1496.

23. Backonja MM, Walk D. QST in peripheral nerve injury pain disorders. Pain. 2012; 153:736-737.

24. Haanpää M, Attal N, Backonja M, Baron R, Bennett M, Bouhassira D, Cruccu G, Hansson P, Haythornthwaite JA, Iannetti GD, Jensen TS, Kauppila T, Nurmikko TJ, et al. NeuPSIG guidelines on neuropathic pain assessment. Pain. 2011; 152:14-27.

25. Rolke R, Magerl W, Campbell KA, Schalber C, Caspari S, Birklein F, Treede RD. Quantitative sensory testing: a comprehensive protocol for clinical trials. Eur J Pain. 2006; 10:77-88.

26. Maier C, Baron R, Tölle TR, Binder A, Birbaumer N, Birklein F, Gierthmühlen J, Flor H, Geber C, Huge V, Krumova EK, Landwehrmeyer GB, Magerl W, et al. Quantitative sensory testing in the German Research Network on Neuropathic Pain (DFNS): somatosensory abnormalities in 1236 patients with different neuropathic pain syndromes. Pain. 2010; 150:439-450.

27. Ti Y, Xie G, Wang Z, Bi X, Ding W, Wang J, Jiang GH, Bu PL, Zhang Y, Zhong M, Zhang W. TRB3 gene silencing alleviates diabetic cardiomyopathy in a type 2 diabetic rat model. Diabetes. 2011; 60:2963-2974.

28. Bennett GJ. Neuropathic pain: an overview. In: Borsook D, ed. Molecular Neurobiology of Pain. Seattle, Washington: IASP Press. 1997; 109-113. 